\title{
Effects of intravenous and inhalation anesthesia on blood glucose and complications in patients with type 2 diabetes mellitus: study protocol for a randomized controlled trial
}

\author{
Xing-Hui Xiong", Chan Chen", Hai Chen, Rui Gao, Qian-Yao Deng, Xing-Wei Cai, Peng Liang, Tao Zhu \\ Department of Anesthesiology and Translational Neuroscience Center, West China Hospital, Sichuan University, Chengdu, China \\ Contributions: (I) Conception and design: C Chen, P Liang; (II) Administrative support: T Zhu; (III) Provision of study materials or patients: C Chen, \\ P Liang, H Chen; (IV) Collection and assembly of data: QY Deng, XW Cai, XH Xiong; (V) Data analysis and interpretation: XH Xiong, R Gao; (VI) \\ Manuscript writing: All authors; (VII) Final approval of manuscript: All authors. \\ \#These authors contributed equally to this work. \\ Correspondence to: Chan Chen; Peng Liang. Department of Anesthesiology and Translational Neuroscience Center, West China Hospital, Sichuan \\ University, No. 37 Guoxue Xiang, Wuhou District, Chengdu, China. Email: xychenchan@gmail.com; liangpeng@wchscu.cn.
}

Background: Diabetes mellitus (DM) is a metabolic disorder syndrome caused by relative or absolute lack of insulin and varying degrees of insulin resistance. The type and regimen of anesthesia may affect perioperative hyperglycemia following major surgical stress. The effect of perioperative anesthetics on the blood glucose level of diabetic patients will play an essential role in the postoperative recovery of patients. However, there is no rigorously-designed randomized controlled trial to compare the effects of total intravenous anesthesia (TIVA) and total inhalation anesthesia (TIHA) on blood glucose and complications in type 2 diabetes. Hence, we design this clinical trial to compare the effects of TIVA and TIHA of hyperglycemia and clinical outcomes in type 2 diabetes undergoing surgery.

Methods: This is a randomized, double-blind, parallel controlled trial. One hundred twelve patients with type 2 DM who meet the qualification criteria will be randomly divided into two groups: TIVA group and TIHA group. The levels of serum insulin and cortisol will be measured before and after the operation, and the levels of blood glucose at different setting time will be monitored. All patients will be followed up by blinded evaluators at baseline and 1, 3, 7, and 30 days after the intervention. The follow-up included postoperative complications [such as myocardial infarction (MI), stroke, renal failure, anastomotic fistula, stress ulcer, incision infection, lung infection] and adverse events.

Discussion: The routinely used clinical anesthesia schemes are TIVA, TIHA and intravenous-inhalation combined anesthesia. We expect that the results of this trial will provide high-quality clinical evidence for the choice of anesthesia options for patients with type $2 \mathrm{DM}$.

Trial registration: Chinese Clinical Trial Registry: ChiCTR2000029247, registration date: 20 January 2020.

Keywords: Diabetes mellitus (DM); intravenous anesthesia; inhalation anesthesia; blood glucose; randomized double-blind controlled trial

Submitted Mar 01, 2020. Accepted for publication Jun 08, 2020.

doi: $10.21037 / \mathrm{atm}-20-2045 \mathrm{a}$

View this article at: http://dx.doi.org/10.21037/atm-20-2045a 


\section{Introduction}

Diabetes mellitus (DM) is a metabolic disorder characterized by a relative or absolute lack of insulin, accompanied by varying degrees of insulin resistance. In China, the overall prevalence of diabetes was $10.9 \%$ and the prevalence of prediabetes was $35.7 \%$ (1). The later stage of diabetes will cause lots of complications, such as diabetic peripheral neuropathy, diabetic vascular disease, renal function damage, limb necrosis, cardio-cerebrovascular disease. Mortality associated with a history of diabetes, stroke, or myocardial infarction (MI) was similar for each condition. Due to any combination of these conditions was associated with multiplicative mortality risk, life expectancy was substantially shorter in people with multimorbidity (2). The life expectancy of stroke, MI and diabetes was shortened at 40 years old and later. However, more and more patients with diabetes need to undergo surgery for the rest of their lives, especially some major abdominal surgery, such as liver surgery, gastrointestinal surgery, and biliary surgery.

During the operation of diabetic patients, anesthesia and surgery can aggravate their condition. Patients with poorly blood glucose controlled may have serious complications such as ketoacidosis, circulatory failure, postoperative infectious complications and even death. The perioperative mortality of patients with diabetes or preoperative hyperglycemia is higher than that of patients without diabetes or hyperglycemia (1,3-7). Decreasing glucose variability during admission for surgery is essential for patients with type 2 diabetes to reducing re-admission rates and length of stay. Glycated hemoglobin (HBA1c) may also identify patients at higher risk of postoperative complications and the possibility of re-admission (8). Regular preoperative inpatient blood glucose monitoring and management can improve intraoperative and postoperative glycemic control and reduce the incidence of hypoglycemia. These changes may eventually improve clinical outcomes (9). To meet the needs of surgery, patients have to be anesthetized, and often need to choose general anesthesia. To ensure that patients can pass through the perioperative period smoothly and safely, we need to choose appropriate anesthetic drugs and anesthetic protocols. The protocols of general anesthesia include total intravenous anesthesia (TIVA), total inhalation anesthesia (TIHA) and intravenous-inhalation combined anesthesia. The effect of perioperative anesthetics on the blood glucose level of diabetic or non-diabetic patients will play an essential role in the postoperative recovery of patients. In patients with unsatisfactory blood glucose control, the wound often does not heal or even necrosis due to the insufficient strength of new tissue and infection (7,10-13). Moreover, diabetes and acute perioperative hyperglycemia may be associated with an increased risk for perioperative neurocognitive disorder (PND) (14,15).

At present, intravenous sedatives or inhaled drugs combined with opioid analgesics and muscle relaxants are commonly used during the operation. Benzodiazepines, an intravenous tranquilizer, can reduce the secretion of cortisol and insulin. Some studies have shown that volatile anesthetics impair glucose tolerance and inhibit insulin secretion by inhibiting ATP-sensitive $\mathrm{K}^{+}$channels on $\beta$ cells, resulting in perioperative hyperglycemia (16-21). Other studies have shown that high-dose propofol infusion can reduce the levels of blood glucose and norepinephrine, thus reducing perioperative blood glucose levels $(22,23)$. Animal experiments have shown that volatile anesthetics can cause hyperglycemia in fed rats $(20,24)$. Subsequently, clinical trials on general patients have shown that compared with the intravenous anesthetic, volatile anesthetic is more likely to increase the incidence rate of intraoperative and postoperative hyperglycemia, while lower insulin levels $(25,26)$. The latest retrospective study shows that sevoflurane and propofol are comparable to the incidence and clinical results of perioperative hyperglycemia in patients with type 2 diabetes undergoing lung surgery (26). There still lack of high-quality clinical evidence related to the choice of anesthesia schemes in type 2 diabetes. It is necessary to design a prospective randomized controlled study to compare the effects of two options of general anesthesia on blood glucose and complications in patients with type $2 \mathrm{DM}$.

The stress response caused by operation can lead to sympathetic nerve excitation, then leads to the increase in the secretion of catecholamine, cortisol, and glucagon in the blood, which promotes gluconeogenesis and glycogen decomposition, eventually leads to the increased level of blood glucose. At the same time, it may lead to insulin resistance and impaired insulin signal to further increase blood glucose (27). A large number of studies have shown that perioperative hyperglycemia is associated with poor prognosis, including wound infection, pneumonia, and cardiovascular events $(27,28)$. Effective control of blood glucose is beneficial to reduce postoperative mortality, hospitalization time and postoperative complications $(9,29)$. The effects of anesthetics on surgical stress, such 
as inhibiting the increase level of blood glucose and glucocorticoids, to reduce the incidence of postoperative incision infection, anastomotic leakage and digestive tract ulcers, and improve the prognosis of patients with diabetes.

In summary, this study focuses on comparing the effects of TIVA with propofol and TIHA with desflurane on blood glucose, cortisol, insulin and complications in type $2 \mathrm{DM}$ patients. The aim of study is to find a suitable anesthetic option to improve the intraoperative management of diabetic patients in clinical. We present the following article in accordance with the SPIRIT reporting checklist (available at http://dx.doi.org/10.21037/atm-20-2045a).

\section{Methods}

\section{Study design}

In this study, a randomized, double-blind, single-center, parallel-controlled clinical trial will be conducted to compare the effects of TIVA and TIHA on patients with type 2 diabetes. A total of 112 patients with type 2 diabetes who are ready for surgery will be randomly divided into the TIVA group and TIHA group. West China Hospital of Sichuan University will be responsible for the recruitment, screening, intervention and follow-up of all patients, and assess all outcomes. Management of the randomization sequence, blinding, and data analyses will be carried out by the Center for Evidence Based West China Hospital, Sichuan University. This protocol is in accordance with the Standard Protocol Items: Recommendations for Interventional Trials (SPIRIT) 2013 statement (30,31). We followed the SPIRIT checklist to address the recommended items in our clinical trial protocol and documents (31). Ethics approval has been received from West China Hospital, Sichuan University. Approved No. of Ethics Committee: 2019-928. Informed written consent will be obtained from all participants (https://cdn.amegroups.cn/ static/public/atm-20-2045a-Supplementary1.pdf). The Case Report Form is available (https://cdn.amegroups.cn/ static/public/atm-20-2045a-Supplementary2.pdf). The trial flow chart is shown in Figure 1, and the flow diagram of participants is given in Table 1 .

\section{Participants}

We will recruit participants between the ages of 18 and 90 with type 2 diabetes who plan to undergo general surgical operation. Potentially qualified participants are being identified by screening the admission records of patients in West China Hospital. According to the inclusion and exclusion criteria, the patient screening will be conducted by one of the authors.

\section{Inclusion criteria}

Participants who fulfill the following criteria could be included: (I) a confirmed diagnosis of type 2 diabetes mellitus; (II) male or female 18 to 90 years of age; (III) American Society of Anesthesiologists class I, II, or III; (IV) general surgery, operation time more than 2 hours; (V) correctly understanding Chinese and express their wishes; (VI) provision of written informed consent.

\section{Exclusion criteria}

The exclusion criteria were as follows: (I) with a history of severe systemic diseases, metabolic disorders, diabetic ketoacidosis or hyperglycemia, diabetic neuropathy, diabetic nephropathy, hepatorenal dysfunction, and neuromuscular disease; (II) with pancreatic cancer, islet cell tumor; (III) being not suitable for the patients according to the doctors or surgeons; (IV) inability to complete research questionnaires; and (V) rejection of randomization.

\section{Sample size}

The sample size calculation was based on a retrospective reference of a similar intervention in similar patient groups using the same primary outcome instrument (26). Blood glucose levels are closely related to the inhibition of stress response by different anesthesia options. According to previous research (26), the mean and standard deviation of blood glucose levels were $(197.5 \pm 40.0)$ in the propofol group and $(212.1 \pm 44.0)$ in the sevoflurane group. The sample size was calculated based on the formula:

$$
n_{1}=n_{2}=2 \frac{\left(Z_{\alpha}+Z_{\beta}\right)^{2} \sigma^{2}}{\delta^{2}}
$$

with a type I error of $5 \%(\alpha=0.05)$ and $80 \%$ power $(\beta=0.1)$. A total of 51 patients are required in each group to show statistical differences between the groups. We estimated that $10 \%$ of patients would drop out of the study; thus, 112 patients have to be included as the sample size. 


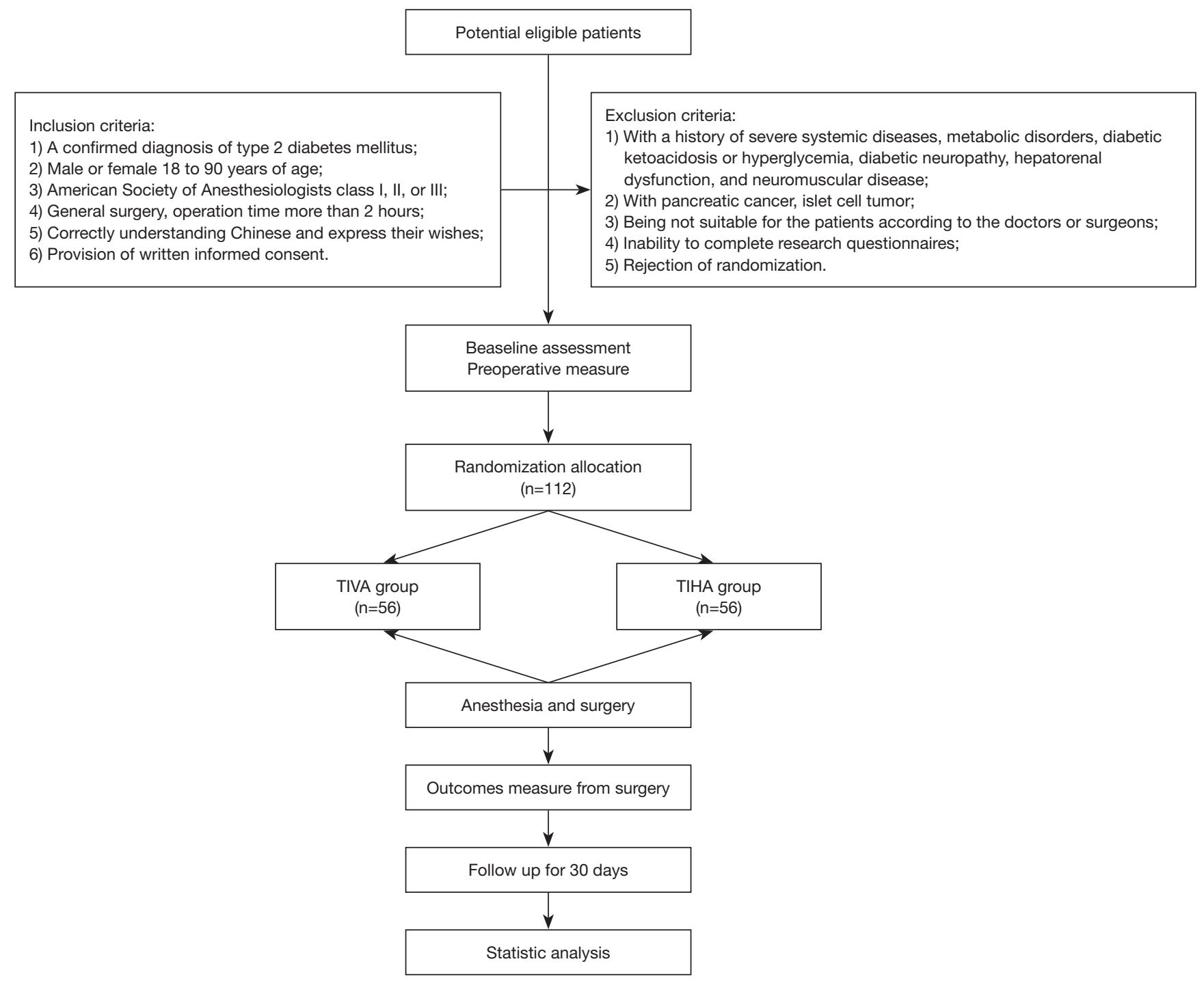

Figure 1 Flow diagram of participants

\section{Randomization and allocation}

The randomization schedule will be developed by the evidence-based medicine center of West China Hospital, Sichuan University. The specific randomization lists will be generated by using the IBM SPSS statistics 26.0 software scheduler computer, and hidden from the screeners, assessors, and participants by a designer from the EvidenceBased Medicine Center who was not involved in the study. Cards with group allocations and detailed intervention plans will be prepared by the Evidence-Based Medicine Center and sealed in an opaque envelope. These sealed envelopes will be marked with the participants' serial number and kept by the screener who is responsible for screening the participants. When a patient has been recruited, the envelope corresponding to the patient's serial number will be sent to the anesthesiologist. The anesthesiologist will open the envelope and perform the intervention according to the anesthesia scheme written on the card (TIVA or TIHA). The information on the allocation list will be kept confidential and kept by anesthesiologists who are not involved in the recruitment of participants or the assessment of outcomes.

\section{Blinding}

The outcome evaluators will be blinded to the randomization 
Table 1 Schedule of recruitment, interventions, and assessments

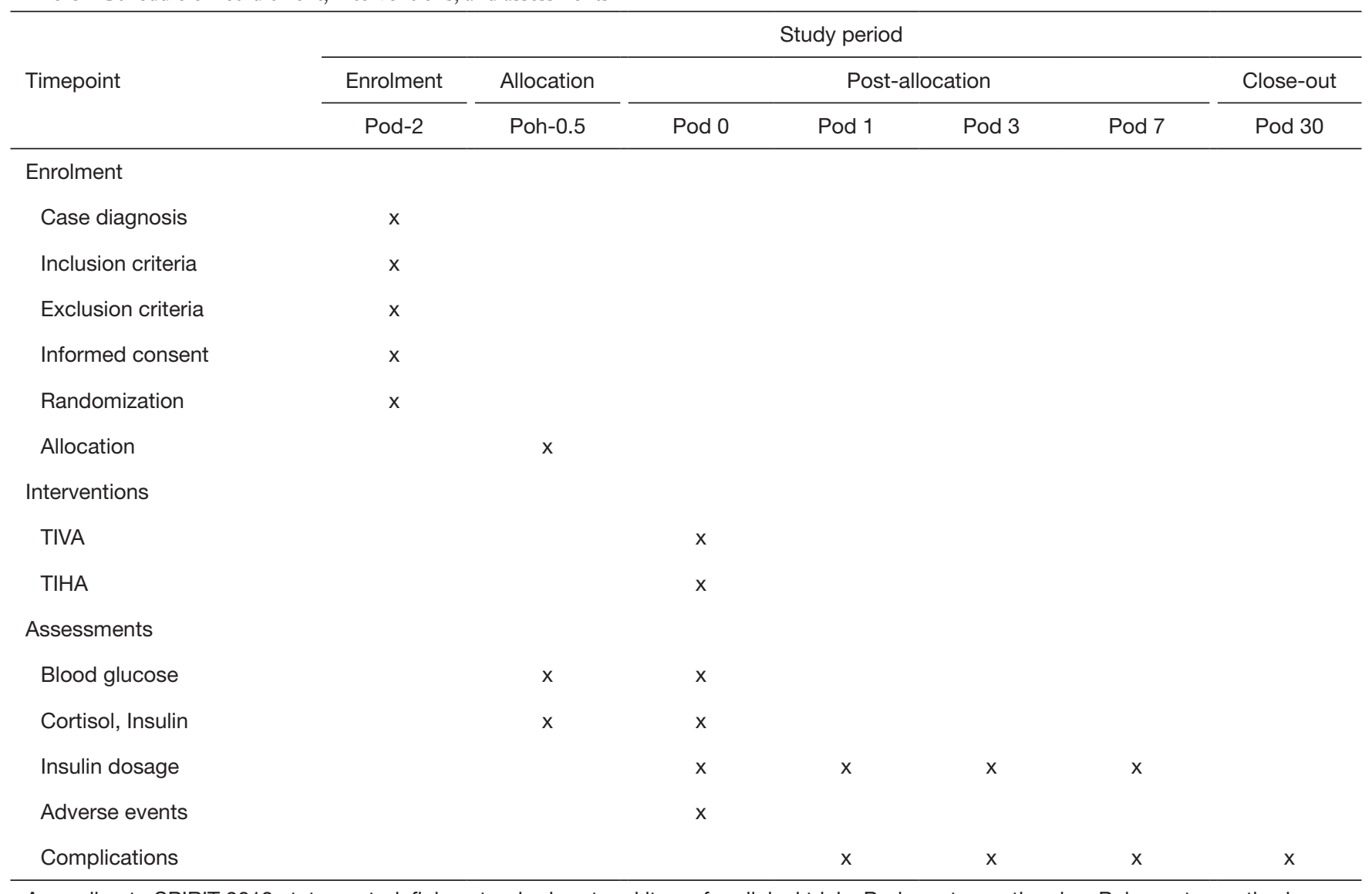

According to SPIRIT 2013 statement: defining standard protocol items for clinical trials. Pod, postoperative day; Poh, postoperative hour.

allocation and will not be involved in the implement of the interventions. Participants will be blinded to the group allocation and will not know whether they have been anesthetized with total intravenous anesthetics or total inhalation anesthetics. It will be impossible to blind the anesthesiologists because they will perform the interventional schemes. However, Anesthesiologists will not participate in the measurement or statistical analysis of outcomes and will be required not to reveal details of their anesthetic procedures to outcome evaluators or participants. The statistician will be blinded to the group allocation until completion of the statistical analyses.

\section{Interventions}

The routinely used clinical anesthesia methods are TIVA, TIHA and intravenous-inhalation combined anesthesia. According to the results of previous studies, we hypothesized that the TIVA group had a more positive effect on blood glucose and complications in patients with type 2 diabetes than the TIHA group. The TIVA group will be maintained by intravenous anesthetic during the operation while the inhalation anesthetic will be used in the TIHA group. If the intraoperative insulin level is more than $10 \mathrm{mmol} / \mathrm{L}$, the $0.1 \mu / \mathrm{kg}$ loading dose of short-acting insulin should be given first, and then continuously pumped according to $0.1 \mu / \mathrm{kg} / \mathrm{h}$.

\section{TIVA}

The patients in the TIVA group will be anesthetized by TIVA. After the patients entering the operating room, the blood glucose at the fingertips will be measured. According to the condition of the patients, the competent anesthesiologist will inject midazolam $0.04 \mathrm{mg} / \mathrm{kg}$, cis atracurium $0.2 \mathrm{mg} / \mathrm{kg}$, sufentanil $0.4 \mu \mathrm{g} / \mathrm{kg}$ and propofol $2 \mathrm{mg} / \mathrm{kg}$ to make the patients be anesthetized, then endotracheal intubation will be performed after mask- 
assisted ventilation for 3 minutes. After successful endotracheal intubation, propofol is continuously pumped at the dose of $4-12 \mathrm{mg} / \mathrm{kg} / \mathrm{min}$, and the dose range of remifentanil is $0.12-0.24 \mu \mathrm{g} / \mathrm{kg} / \mathrm{min}$ during surgery. The anesthesiologist will adjust the drug dose according to the surgical stimulation condition, vital signs of the patient, and bispectral index of EEG (BIS).

The monitoring of the BIS will be performed in all patients. The BIS score ranges from 40 to 60 during operation while which ranges from 60 to 70 during skin suture. All patients will adopt volume-controlled breathing mode: tidal volume can be controlled during $6-8 \mathrm{~mL} / \mathrm{kg}$, respiratory frequency ranges from 12 to 14 times/min, inspiratory/ expiratory ratio is $1: 2$, and end-tidal carbon dioxide (PETCO2) will be controlled from 35 to $45 \mathrm{mmHg}$. The mean arterial pressure and heart rate are maintained within the range of $\pm 20 \%$, and vasoactive drugs are used if necessary.

\section{TIHA}

The anesthesia induction process in the inhalation group is the same as that in the TIVA group. Desflurane combined with remifentanil will be used to maintain anesthesia after induction. The anesthesiologist will adjust the dose of anesthetics according to the surgical condition and the patient's vital signs. The intraoperative monitoring and treatment in the inhalation group is the same as those in the TIVA group.

\section{Intervention regimen}

During the entire surgical procedure, the patient will be anesthetized by inhalation or intravenous anesthesia until the patient wakes up at the end of the operation. All inhaled anesthesia will use the anesthetic machine produced by Ohmeda in the United States, and all intravenous anesthesia will use the medical microinjection pump compacts in Belan, Germany.

\section{Follow-up period}

During the 30-day follow-up period, participants with blood glucose levels above $10 \mathrm{mmol} / \mathrm{L}$ will be given subcutaneous insulin therapy. At post-operation day (POD) 1, 3, 7, and 30 , the outcome assessor will investigate the postoperative complications by telephone follow-up.

\section{Outcome measurement}

The following baseline descriptive data will be obtained through questionnaire and physical examination: age, gender, marital status, education level, employment status, disease history and complications of diabetes, history of drug use, past health problems. Table 1 shows a summary of all the measures in the trial.

\section{Primary outcome measures}

The primary outcomes are the blood glucose levels at different setting time, including preoperative, immediate intubation, skin incision, $1 \mathrm{~h}, 2 \mathrm{~h}$ after the operation start, 1 and $2 \mathrm{~h}$ after the operation.

\section{Secondary outcome measures}

The secondary outcome measures are serum insulin and cortisol levels $30 \mathrm{~min}$ before and $30 \mathrm{~min}$ after the operation. The participants' incidence of complications, including MI, stroke, renal failure, anastomotic fistula, stress ulcer, incision infection, and pulmonary infection, will be followed up postoperatively. The complications will be recorded by telephone investigation during the follow-up period.

\section{Adverse events and safety}

Any adverse events (defined as any functional lesion caused by the interventions, such as anesthetic allergy, poorly anesthetic effect during the operation, and so on) will be recorded. If any adverse event occurs, the doctor will provide the corresponding treatment to the patient, change, or even stop the anesthesia protocol. The adverse events will be immediately reported to the primary investigator and ethics committee to decide if the patient needs to withdraw from the trial.

\section{Data collection and management}

The demographic and baseline characteristic data will be collected by screeners when the patients are recruited. Outcome assessors will measure clinical outcome measurement after the operation is completed. During the follow-up period, the participants' incidence of complications will be investigated by a telephone call at 1,3 , 7 , and 30 days after the operation. 
The researchers will control the quality of data collection and be responsible for data entry. The data administrator will be responsible for cleaning, identifying, encoding, and converting the initial data to the appropriate format for data analysis.

\section{Statistical analysis}

All outcomes will undergo an intent-to-treat (ITT) analysis based on the initial anesthesia allocation. The missing value will be filled up by last-observation-carried-forward method. Analyses will be performed using IBM SPSS 26 (version 26.0, IBM Corp., New York, NY, USA) software. For continuous variables and descriptive values, means, standard deviations will be reported. For the enumerative variables, frequency and corresponding percentage will be given. For the variables with a normal distribution, statistical comparisons between the groups will be made by using a $t$-test. If the variables have a non-normal distribution or ordinal level, statistical comparison between groups will be made using the Mann-Whitney $U$ test. Measures with a discrete distribution will be expressed as percentages and analyzed by the chi-squared test or Fisher's exact test as appropriate. The total amount of additional insulin used between two groups will be analyzed.

\section{Discussion}

In this randomized, double-blind controlled trial of patients with type 2 diabetes, we intend to compare the effects of TIVA and TIHA on blood glucose and complications in patients with type 2 diabetes. We will conduct this trial in strict accordance with the Consolidated Standards of Reporting Trials (CONSORT) (32), and will be able to determine the effectiveness of general anesthesia. First of all, we estimate the optimal sample size to ensure adequate test performance. Second, we ensure that this study is a genuinely randomized controlled trial through the full implementation of randomization and blindness. TIVA and TIHA are two routine anesthetic schemes commonly used in the clinic. The alternative anesthesia is intravenous-inhalation combined anesthesia, but it is uncontrollable, so this anesthetic method is not adopted. However, the method of anesthesia can't set blindness to the anesthesiologist who implement the intervention, so it can only set blindness to participants and followers, there is a particular risk of subjective bias. In the evaluation index, a reasonable specification and a rigorous professional scale are designed as the assessment index of the primary outcomes, so as to avoid the subjective assessment and reduce the data distortion and deviation. Anesthesiologists with abundant clinical experience will perform the intervention (TIVA or TIHA). This will improve the compliance of participants and reduce the risk of loss of follow-up.

We hypothesized that intravenous anesthesia had a positive effect on blood glucose and complications in patients with type 2 diabetes compared with inhalation anesthesia. Inhalation anesthetics lead to impaired glucose tolerance and insulin secretion by inhibiting ATPsensitive $\mathrm{K}^{+}$channels on $\beta$ cells, resulting in perioperative hyperglycemia $(21,24)$. Other studies have shown that high-dose propofol infusion can reduce blood glucose and norepinephrine levels, thus decreasing perioperative blood glucose levels in non-diabetes patients $(22,23)$. However, for patients with diabetes, who are relative or absolute lack of insulin and varying degrees of insulin resistance, there are no rigorous randomized controlled studies on the changes of blood glucose caused by the above two anesthetic methods.

In summary, we combined the appropriate clinical trial design with the characteristics of anesthesia to complete the scientific design. We will report the results of clinical trials in accordance with international norms for randomized controlled trial reporting (COSORT) $(32,33)$. We expect that the results of this trial will provide scientific and rigorous clinical evidence for the option of general anesthesia schemes for patients with type $2 \mathrm{DM}$.

\section{Acknowledgments}

Funding: This work was supported by the National Natural Science Foundation of China (grant numbers 81870858 , 81500937), the National Natural Science Foundation of China (grant number 81671062), and the National Key R\&D Program of China (grant number 2018YFC2001800).

\section{Footnote}

Reporting Checklist: The authors have completed the SPIRIT reporting checklist. Available at http://dx.doi.org/10.21037/ atm-20-2045a

Conflicts of Interest: All authors have completed the ICMJE uniform disclosure form (available at http://dx.doi. org/10.21037/atm-20-2045a). The authors have no conflicts of interest to declare. 


\section{Page 8 of 9}

Ethical Statement: The authors are accountable for all aspects of the work in ensuring that questions related to the accuracy or integrity of any part of the work are appropriately investigated and resolved. The study was conducted in accordance with the Declaration of Helsinki (as revised in 2013). The study protocol was approved by Medical Ethics Committee of West China Hospital, Sichuan University. Informed consent will be obtained from each patient. Blood samples will be collected with the participants' consent. The results will be disseminated to the public through papers in open access journals.

Open Access Statement: This is an Open Access article distributed in accordance with the Creative Commons Attribution-NonCommercial-NoDerivs 4.0 International License (CC BY-NC-ND 4.0), which permits the noncommercial replication and distribution of the article with the strict proviso that no changes or edits are made and the original work is properly cited (including links to both the formal publication through the relevant DOI and the license). See: https://creativecommons.org/licenses/by-nc-nd/4.0/.

\section{References}

1. Wang L, Gao P, Zhang M, et al. Prevalence and Ethnic Pattern of Diabetes and Prediabetes in China in 2013. JAMA 2017;317:2515-23.

2. Emerging Risk Factors Collaboration, Di Angelantonio E, Kaptoge S, et al. Association of Cardiometabolic Multimorbidity With Mortality. JAMA 2015;314:52-60.

3. Kotagal M, Symons RG, Hirsch IB, et al. Perioperative hyperglycemia and risk of adverse events among patients with and without diabetes. Ann Surg 2015;261:97-103.

4. Yeh CC, Liao CC, Chang YC, et al. Adverse outcomes after noncardiac surgery in patients with diabetes: a nationwide population-based retrospective cohort study. Diabetes care 2013;36:3216-21.

5. Underwood P, Askari R, Hurwitz S, et al. Preoperative $\mathrm{A} 1 \mathrm{C}$ and clinical outcomes in patients with diabetes undergoing major noncardiac surgical procedures. Diabetes care 2014;37:611-6.

6. Subramaniam B, Lerner A, Novack V, et al. Increased glycemic variability in patients with elevated preoperative HbA1C predicts adverse outcomes following coronary artery bypass grafting surgery. Anesth Analg 2014;118:277-87.

7. Shanks AM, Woodrum DT, Kumar SS, et al. Intraoperative hyperglycemia is independently associated with infectious

\section{Xiong et al. TIVA vs. TIHA on blood glucose in type 2 diabetes}

complications after non-cardiac surgery. BMC Anesthesiol 2018;18:90.

8. Hsieh CJ. High Glucose Variability Increases 30-Day Readmission Rates in Patients with Type 2 Diabetes Hospitalized in Department of Surgery. Sci Rep 2019;9:14240.

9. Garg R, Schuman B, Bader A, et al. Effect of Preoperative Diabetes Management on Glycemic Control and Clinical Outcomes After Elective Surgery. Ann Surg 2018;267:858-62.

10. Karimian N, Niculiseanu P, Amar-Zifkin A, et al. Association of Elevated Pre-operative Hemoglobin A1c and Post-operative Complications in Non-diabetic Patients: A Systematic Review. World J Surg 2018;42:61-72.

11. McConnell YJ, Johnson PM, Porter GA. Surgical site infections following colorectal surgery in patients with diabetes: association with postoperative hyperglycemia. J Gastrointest Surg 2009;13:508-15.

12. Long CA, Fang ZB, Hu FY, et al. Poor glycemic control is a strong predictor of postoperative morbidity and mortality in patients undergoing vascular surgery. J Vasc Surg 2019;69:1219-26.

13. Margonis GA, Amini N, Sasaki K, et al. Perioperative Hyperglycemia and Postoperative Outcomes in Patients Undergoing Resection of Colorectal Liver Metastases. J Gastrointest Surg 2017;21:228-37.

14. Hermanides J, Qeva E, Preckel B, et al. Perioperative hyperglycemia and neurocognitive outcome after surgery: a systematic review. Minerva Anestesiol 2018;84:1178-88.

15. Zhang X, Yan X, Gorman J, et al. Perioperative hyperglycemia is associated with postoperative neurocognitive disorders after cardiac surgery. Neuropsychiatr Dis Treat 2014;10:361-70.

16. Tanaka T, Nabatame H, Tanifuji Y. Insulin secretion and glucose utilization are impaired under general anesthesia with sevoflurane as well as isoflurane in a concentrationindependent manner. J Anesth 2005;19:277-81.

17. Lattermann R, Schricker T, Wachter U, et al. Understanding the mechanisms by which isoflurane modifies the hyperglycemic response to surgery. Anesth Analg 2001;93:121-7.

18. Tanaka K, Kawano T, Tomino T, et al. Mechanisms of impaired glucose tolerance and insulin secretion during isoflurane anesthesia. Anesthesiology 2009;111:1044-51.

19. Tanaka K, Kawano T, Tsutsumi Y, et al. Differential effects of propofol and isoflurane on glucose utilization and insulin secretion. Life Sci 2011;88:96-103.

20. Kitamura T, Ogawa M, Kawamura G, et al. The effects 
of sevoflurane and propofol on glucose metabolism under aerobic conditions in fed rats. Anesth Analg 2009; 109:1479-85.

21. Saho S, Kadota Y, Sameshima T, et al. The effects of sevoflurane anesthesia on insulin secretion and glucose metabolism in pigs. Anesth Analg 1997;84:1359-65.

22. Cok OY, Ozkose Z, Pasaoglu H, et al. Glucose response during craniotomy: propofol-remifentanil versus isofluraneremifentanil. Minerva Anestesiol 2011;77:1141-8.

23. Ihn CH, Joo JD, Choi JW, et al. Comparison of stress hormone response, interleukin-6 and anaesthetic characteristics of two anaesthetic techniques: volatile induction and maintenance of anaesthesia using sevoflurane versus total intravenous anaesthesia using propofol and remifentanil. J Int Med Res 2009;37:1760-71.

24. Zuurbier CJ, Keijzers PJ, Koeman A, et al. Anesthesia's effects on plasma glucose and insulin and cardiac hexokinase at similar hemodynamics and without major surgical stress in fed rats. Anesth Analg 2008;106:135-42, table of contents.

25. Wu Y, Zhang L, Yin G, et al. Stress Response to Propofol versus Isoflurane Anesthesia in Patients Undergoing Gastric Surgery. J Coll Physicians Surg Pak 2019;29:201-4.

26. Kim H, Han J, Jung SM, et al. Comparison of sevoflurane and propofol anesthesia on the incidence of hyperglycemia in patients with type 2 diabetes undergoing lung surgery.

Cite this article as: Xiong $\mathrm{XH}$, Chen $\mathrm{C}$, Chen $\mathrm{H}$, Gao R, Deng QY, Cai XW, Liang P, Zhu T. Effects of intravenous and inhalation anesthesia on blood glucose and complications in patients with type 2 diabetes mellitus: study protocol for a randomized controlled trial. Ann Transl Med 2020;8(13):825. doi: 10.21037/atm-20-2045a
Yeungnam Univ J Med 2018;35:54-62.

27. Finnerty CC, Mabvuure NT, Ali A, et al. The surgically induced stress response. JPEN J Parenter Enteral Nutr 2013;37:21S-9S.

28. Palermo NE, Gianchandani RY, McDonnell ME, et al. Stress Hyperglycemia During Surgery and Anesthesia: Pathogenesis and Clinical Implications. Curr Diab Rep 2016;16:33.

29. Simha V, Shah P. Perioperative Glucose Control in Patients With Diabetes Undergoing Elective Surgery. JAMA 2019;321:399-400.

30. Chan AW, Tetzlaff JM, Gotzsche PC, et al. SPIRIT 2013 explanation and elaboration: guidance for protocols of clinical trials. BMJ 2013;346:e7586.

31. Chan AW, Tetzlaff JM, Altman DG, et al. SPIRIT 2013 statement: defining standard protocol items for clinical trials. Ann Intern Med 2013;158:200-7.

32. Moher D, Hopewell S, Schulz K, et al. CONSORT 2010 explanation and elaboration: updated guidelines for reporting parallel group randomised trials. BMJ (Clinical research ed) 2010;340:c869.

33. Hoffmann TC, Glasziou PP, Boutron I, et al. Better reporting of interventions: template for intervention description and replication (TIDieR) checklist and guide. BMJ (Clinical research ed) 2014;348:g1687. 